\title{
Image processing in the semidiscrete group of rototranslations
}

\author{
Dario Prandi ${ }^{1}$, Ugo Boscain ${ }^{2,3}$, Jean-Paul Gauthier ${ }^{3,4}$ \\ 1 CEREMADE, U. Paris Dauphine \\ ${ }^{2}$ CMAP, École Polytechnique, Route de Saclay, 91128 Palaiseau Cedex, France \\ ${ }^{3}$ INRIA Team GECO \\ ${ }^{4}$ LSIS, Université de Toulon USTV, 83957, La Garde Cedex, France
}

\begin{abstract}
It is well-known, since [12], that cells in the primary visual cortex V1 do much more than merely signalling position in the visual field: most cortical cells signal the local orientation of a contrast edge or bar - they are tuned to a particular local orientation. This orientation tuning has been given a mathematical interpretation in a subRiemannian model by Petitot, Citti, and Sarti $[14,6]$. According to this model, the primary visual cortex V1 lifts grey-scale images, given as functions $f: \mathbb{R}^{2} \rightarrow[0,1]$, to functions $L f$ defined on the projectivized tangent bundle of the plane $P T \mathbb{R}^{2}=\mathbb{R}^{2} \times \mathbb{P}^{1}$. Recently, in [1], the authors presented a promising semidiscrete variant of this model where the Euclidean group of rototranslations $S E(2)$, which is the double covering of $P T \mathbb{R}^{2}$, is replaced by $S E(2, N)$, the group of translations and discrete rotations. In particular, in [15], an implementation of this model allowed for state-of-the-art image inpaintings.

In this work, we review the inpainting results and introduce an application of the semidiscrete model to image recognition. We remark that both these applications deeply exploit the Moore structure of $S E(2, N)$ that guarantees that its unitary representations behaves similarly to those of a compact group. This allows for nice properties of the Fourier transform on $S E(2, N)$ exploiting which one obtains numerical advantages.
\end{abstract}

\section{The semi-discrete model}

The starting point of our work is the sub-Riemannian model of the primary visual cortex V1 $[14,6]$, and our recent contributions $[3,1,2,4]$. This model has also been deeply studied in $[8,11]$. In the sub-Riemannian model, V1 is modeled as the projective tangent bundle $P T \mathbb{R}^{2} \cong \mathbb{R}^{2} \times \mathbb{P}^{1}$, whose double covering is the roto-translation group $S E(2)=\mathbb{R}^{2} \rtimes \mathbb{S}^{1}$, endowed with a left-invariant subRiemannian structure that mimics the connections between neurons. In particular, grayscale visual stimuli $f: \mathbb{R}^{2} \rightarrow[0,1]$ feeds V1 neurons $N=(x, \theta) \in P T \mathbb{R}^{2}$ with an extracellular voltage $\mathcal{L} f(\xi)$ that is widely accepted to be given by $\mathcal{L} f(\xi)=\left\langle f, \Psi_{\xi}\right\rangle$. The functions $\left\{\Psi_{\xi}\right\}_{\xi \in P T \mathbb{R}^{2}}$ are the receptive fields. A good fit is $\Psi_{(x, \theta)}=\pi(x, \theta) \Psi$ where $\Psi$ is the Gabor filter (a sinusoidal multiplied by a Gaussian function) and $\pi(x, \theta) \Psi(y):=\Psi\left(R_{-\theta}(x-y)\right)$. 
In this work we consider a slightly different setting, by assuming that neurons are sensible only to a finite (small) number of orientations. This assumption is based on the observation of the organization of the visual cortex in pinwheels: we conjecture that there are topological constraints that prevent the possibility of detecting a continuum of directions even when sending the distance between pinwheels to zero. This assumption leads us to consider the group of translations and discrete rotations $S E(2, N)=\mathbb{R}^{2} \rtimes \mathbb{Z}_{N}$, for some $N \in \mathbb{N}$, where the action of $k \in \mathbb{Z}_{N}$ on $\mathbb{R}^{2}$ is the rotation of angle $2 \pi k / N$.

To be more precise, our model is based on the following assumptions:

1. Grayscale visual stimuli coming from the retina are modeled as functions $f \in L^{2}\left(\mathbb{R}^{2}\right)$

2. The primary visual cortex is modeled as $S E(2, N)$ and its activation patterns as $\varphi \in L^{2}(S E(2, N))$;

3. There exists a linear function $\mathcal{L}: L^{2}\left(\mathbb{R}^{2}\right) \rightarrow L^{2}(S E(2, N))$ that lifts visual stimuli to activation patterns in the primary visual cortex, of the form $\mathcal{L} f(x, k)=\langle f, \pi(x, k) \Psi\rangle$ for some $\Psi \in L^{2}\left(\mathbb{R}^{2}\right)$.

4. An excited neuron activate neighboring neurons according to the SDE

$$
d A_{t}=X_{1} d W_{t}+d \Theta_{t},
$$

where $X_{1}(x, k)=\cos (2 \pi k / N) \partial_{x_{1}}+\sin (2 \pi k / N) \partial_{x_{2}}, W$ is a Wiener process and $\Theta$ is a Poisson jump process on $\mathbb{Z}_{N}$ with jump probability equal to $1 / 2$ on both sides.

Remark 1. Observe that the lift operator $\mathcal{L}$ respects the shift-twist symmetry of V1. (See e.g. [5].) That is, letting $\Lambda$ be the left regular representation of $S E(2, N)$ in $L^{2}\left(S E(2, N)\right.$ ) (i.e., $\left.[\Lambda(x, k) \varphi](y, h)=\varphi\left((x, k)^{-1}(y, h)\right)\right)$ and $\pi$ the quasi-regular representation of $S E(2, N)$ in $L^{2}\left(\mathbb{R}^{2}\right)$ (i.e., $[\pi(x, k) f](y)=$ $\left.f\left(R_{-k}(y-x)\right)\right)$, it holds $\Lambda(x, k) L f=L(\pi(x, k) f)$.

\section{$2 \quad$ Image inpainting}

The algorithm we now present for image inpainting is inspired by the neurophysiological process of modal completion, that is, the perception of a shape even when it is not actually drawn. A famous example is that of the Kanizsa triangle. Our working assumption is that modal completion is caused by the following neurophysiological principle: Corrupted images are reconstructed by the natural diffusion in V1, induced by the SDE (1), which for small times follows the less expensive paths (geodesics) to activate unexcited neurons.

From the practical point of view, images are reconstructed through the following algorithm. For details see [1]. 


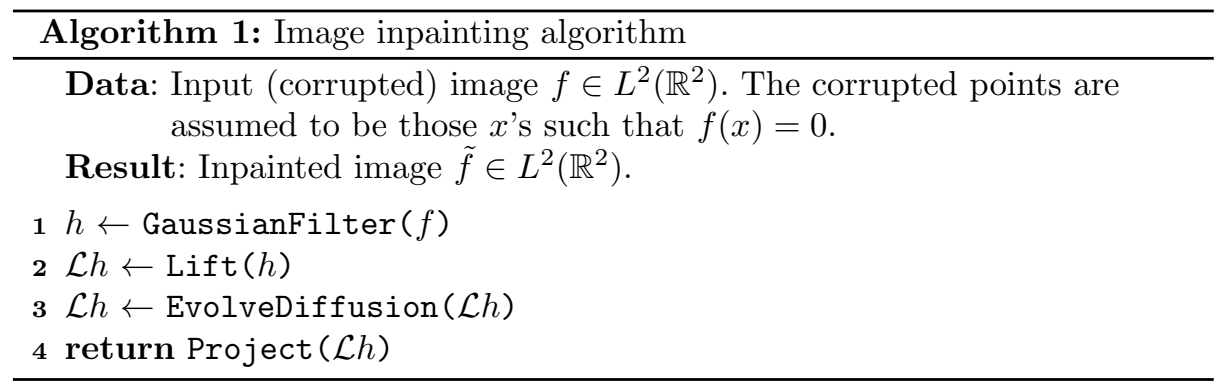

A description of the 4 functions used in Algorithm 1 follows.

1. GaussianFilter: Smooths the input via a Gaussian filter. As explained in [2], the result of this procedure is generically a Morse function (i.e. with isolated non-degenerate critical points only).

2. Lift: Given a Morse function $h$ lifts it to $L h$ defined on $S E(2, N)$, obtained as follows. We let $\theta(x) \in[0, \pi)$ to be orientation of $\nabla h(x)$, when it is well defined. Then, we define $\operatorname{Lh}(x, k)=h(x)$ if $k \cong \theta(x)$ and 0 otherwise Here, the formulation $k \cong \theta(x)$ means that $2 \pi k / N$ is the nearest point to $\theta(x)$ among $\left\{2 \pi \ell / N \mid \ell \in \mathbb{Z}_{N}\right\}$. Since $h$ is a Morse function, $\theta(x)$ is not well defined on isolated points. In this case, we let $L h(x, h):=h(x) / N$ for any $h \in \mathbb{Z}_{N}$.

3. EvolveDiffusion: Given a function $L h$ on $S E(2, N)$ evolves it according to (1). An efficient way to compute this diffusion is presented in [1], and recalled in Algorithm 2.

4. Project: Given a function $\varphi$ on $S E(2, N)$ returns its projection on $\mathbb{R}^{2}$ defined as $P \varphi(x):=\max _{k \in \mathbb{Z}_{N}} \varphi(x, k)$.

Remark 2. The Lift procedure detailed above is not obtained via a convolution with an oriented wavelet as it is supposed to be the case in V1. However, it can be seen as the limit when the support of the wavelet tends to zero and experiments have shown that it yields more precise reconstructions.

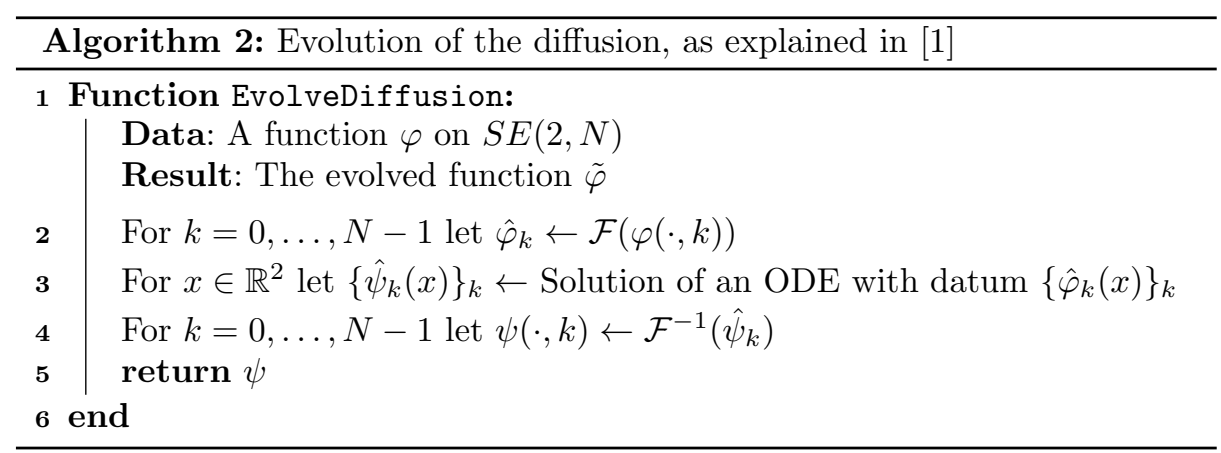

In Figure 1 we present two different inpainting results. While the first one is obtained using Algorithm 1, to produce the second one we added some heuristic procedure (detailed in $[1,15]$ ) in order to prevent the diffusion from modifying the non-corrupted part of the image. 

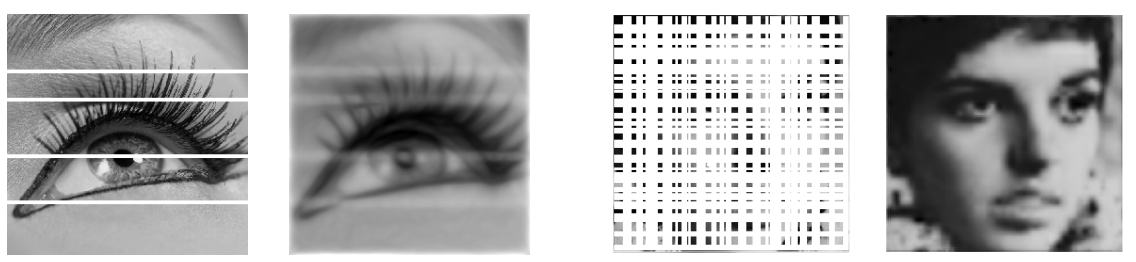

Fig. 1. Two inpaintings.

\section{Image recognition}

The fact that images are lifted to V1, which has the (group) structure of $S E(2, N)$ allows for a natural description of the process of invariant image recognition. (That is, recognizing images under the roto-translation action of $S E(2, N)$.) Namely, we propose to use the bispectrum as an invariant under the action of $S E(2, N)$. These invariants are well established in statistical signal processing [7] and have been introduced and studied in the context of $S E(2, N)$ and of compact groups in [18]. We mention also [13], devoted to the bispectrum on homogeneous spaces of compact groups.

Let us introduce some generalities on the (generalized) Fourier transform on $S E(2, N)$. Since this group is a non-commutative unimodular semi-direct product, computing the Fourier transform of an $L^{2}(S E(2, N))$ requires the knowledge of the (continuous) irreducible unitary representations $T^{\lambda}$ of $S E(2, N)$. Here, $\lambda$ is an index taking values in the dual object of $S E(2, N)$, which is denoted by $S \widehat{E(2, N)}$ and is the set of equivalence classes of irreducible unitary representations. (See, e.g., [10].) Exploiting the semi-direct product structure of $S E(2, N)$, by Mackey machinery this dual can be shown to be the union of the slice $\mathcal{S} \subset \mathbb{R}^{2}$, which in polar coordinates is $\mathbb{R}_{+}^{*} \times[0,2 \pi / N)$, to which we glue $\mathbb{Z}_{N}$ on 0 . Since it is possible to show that to invert the Fourier transform it is enough to consider representations parametrized by $\mathcal{S}$, we will henceforth ignore the $\mathbb{Z}_{N}$ part of the dual. A crucial fact for the following is that $S E(2, N)$ is a Moore group, that is, all the $T^{\lambda}$ act on finite-dimensional spaces, that is $\mathbb{C}^{N}$ for $\lambda \in \mathcal{S}$. This is not true for the roto-translation group $S E(2)$ and is indeed one of the main theoretical advantages of the semi-discrete model.

The matrix-valued Fourier coefficient of a function $\varphi \in L^{2}(S E(2, N)) \cap$ $L^{1}(S E(2, N))$ for $\lambda \in S \widehat{E(2, N)}$ is $\hat{\varphi}\left(T^{\lambda}\right)=\int_{S E(2, N)} f(a) T^{\lambda}\left(a^{-1}\right) d a$. This is essentially the same formula for the Fourier transform on $\mathbb{R}$, which is a scalar and is obtained using the representations $T^{\lambda}(x)=e^{2 \pi i x \lambda}$. As usual, the above formula can be extended to a linear isometry $\mathcal{F}: L^{2}(S E(2, N)) \rightarrow L^{2}(S \widehat{E(2, N)})$. The bispectrum of $\varphi$ is then the quantity

$$
B_{\varphi}\left(\lambda_{1}, \lambda_{2}\right)=\hat{\varphi}\left(T^{\lambda_{1}}\right) \otimes \hat{\varphi}\left(T^{\lambda_{2}}\right) \circ \hat{\varphi}\left(T^{\lambda_{1}} \otimes T^{\lambda_{2}}\right)^{*} \quad \forall\left(\lambda_{1}, \lambda_{2}\right) \in \mathcal{S} .
$$

This quantity can be interpreted as the Fourier transform of the triple correlation function, see [13]. 
In a forthcoming paper we will present (in a more general setting) the following result.

Theorem 1. The bispectral invariants discriminate on the set $\mathcal{G}$ of functions $\varphi \in L^{2}(S E(2, N))$ such that the matrices $\hat{\varphi}\left(T^{\lambda}\right)$ are invertible for a.e. $\lambda \in \mathcal{S}$. That is, $\varphi_{1}, \varphi_{2} \in \mathcal{G}$ are such that $B_{\varphi_{1}}=B_{\varphi_{2}}$ if and only if $\varphi_{1}=\Lambda(x, k) \varphi_{2}$ for some $(x, k) \in S E(2, N)$.

Unfortunately, when considering the lifts of visual stimuli $f \in L^{2}\left(\mathbb{R}^{2}\right)$ under lifts, an easy computation shows that $\widehat{\mathcal{L f}}\left(T^{\lambda}\right)=\overline{\omega_{f}(\lambda)} \otimes \overline{\omega_{\Psi}(\lambda)}$ where $\omega_{f}(\lambda)=$ $\left(\hat{f}\left(R_{-k} \lambda\right)\right)_{k \in \mathbb{Z}_{N}} \in \mathbb{C}^{N}$. This immediately implies that $\operatorname{rank} \widehat{\mathcal{L} f}\left(T^{\lambda}\right) \leq 1$ and hence that range $\mathcal{L} \cap \mathcal{G}=\varnothing$.

Using the previous formula for the Fourier transform of lifted functions and under mild assumptions on the wavelet $\Psi$ one can show that the bispectrum $B\left(\lambda_{1}, \lambda_{2}\right)$ is completely determined by the quantity

$$
I_{f}^{2}\left(\lambda_{1}, \lambda_{2}\right)=\left\langle\omega_{f}\left(\lambda_{1}\right) \odot \omega_{f}\left(\lambda_{2}\right), \omega_{f}\left(\lambda_{1}+\lambda_{2}\right)\right\rangle .
$$

It is still an open question (although we conjecture it to be true) whether the bispectrum discriminates on a "big" set of range $\mathcal{L}$.

To bypass the difficulty posed by the non-invertibility of the Fourier transform for lifted functions, we are led to consider the rotational bispectrum:

$\tilde{B}_{\varphi}\left(\lambda_{1}, \lambda_{2}, k\right):=\hat{\varphi}\left(T^{R_{h} \lambda_{1}}\right) \otimes \hat{\varphi}\left(T^{\lambda_{2}}\right) \circ \hat{\varphi}\left(T^{\lambda_{1}} \otimes T^{\lambda_{2}}\right)^{*} \quad \forall\left(\lambda_{1}, \lambda_{2}\right) \in \mathcal{S}, \forall h \in \mathbb{Z}_{N}$.

Observe that the rotational bispectrum is invariant only under the action of $\mathbb{Z}_{N} \subset S E(2, N)$ but not under translations. To avoid this problem, let us consider the set $\mathcal{A} \subset L^{2}\left(\mathbb{R}^{2}\right)$ of compactly supported functions with non-zero average ${ }^{5}$. We can then define the barycenter $c_{f} \in \mathbb{R}^{2}$ of $f \in \mathcal{A}$ as

$$
c_{f}=\frac{1}{\operatorname{avg} f}\left(\int_{\mathbb{R}^{2}} x_{1} f(x) d x, \int_{\mathbb{R}^{2}} x_{2} f(x) d x\right), \quad j=1,2,
$$

and the centering operator $\Phi: \mathcal{A} \rightarrow \mathcal{A}$ as $\Phi f(x):=f\left(x-c_{f}\right)$. Then, considering the lift $\mathcal{L}_{c}=\mathcal{L} \circ \Phi$, we have that $\mathcal{L}_{c} f=\mathcal{L}_{c} g$ if and only if $g$ is a translate of $f$.

Finally, we have the following.

Theorem 2. Let $\mathcal{R} \subset L^{2}\left(\mathbb{R}^{2}\right)$ be the set of compactly supported functions $f$ such that

1. $\hat{f}(\lambda) \neq 0$ for a.e. $\lambda \in \mathbb{R}^{2}$;

2. the circulant matrix associated with $\omega_{f}(\lambda)$ is invertible for a.e. $\lambda \in \mathbb{R}^{2}$.

Then, if $\Psi \in \mathcal{R}$ the rotational bispectrum discriminates on $\mathcal{L}_{c}(\mathcal{R} \cap \mathcal{A})$. That is, for any $f, g \in \mathcal{R} \cap \mathcal{A}$ it holds that $\tilde{B}_{\mathcal{L}_{c} f}=\tilde{B}_{\mathcal{L}_{c} g}$ if and only if $f=\pi(x, k) g$ for some $(x, k) \in S E(2, N)$.

Moreover, since set $\mathcal{R}$ is residual in the compactly supported functions $L^{2}\left(\mathbb{R}^{2}\right)$, the rotational bispectrum is generically discriminating on the compactly supported functions of $L^{2}\left(\mathbb{R}^{2}\right)$.

\footnotetext{
${ }^{5}$ Recall that the average of $f \in L^{1}\left(\mathbb{R}^{2}\right)$ is avg $f=\int_{\mathbb{R}^{2}} f(x) d x$.
} 
Let us observe that, if $\Psi \in \mathcal{R}$, then $\tilde{B}_{\mathcal{L}_{c} f}\left(\lambda_{1}, \lambda_{2}, k\right)$ is completely determined by the quantities

$$
I_{f}^{2}\left(\lambda_{1}, \lambda_{2}, k\right)=\left\langle\omega_{\Phi f}\left(R_{k} \lambda_{1}\right) \odot \omega_{\Phi f}\left(\lambda_{2}\right), \omega_{\Phi f}\left(\lambda_{1}+\lambda_{2}\right)\right\rangle .
$$

In particular, computing the rotational bispectrum requires $N$ times more operations than computing the bispectrum.

\subsection{Implementation and numerical experiments}

We now describe how to efficiently compute the bispectrum invariants ${ }^{6}$. The same method, with the obvious modifications, also works for the computation of the rotational bispectrum. We then show that the difference in norm of the bispectrum and the rotational bispectrum strongly separates images of different objects. The next natural step, that we will tackle in a forthcoming paper, is to use these invariants in machine learning algorithms as SVM's or AdaBoosts.

As previously remarked, to compute the bispectrum invariants it is enough to compute the quantities $I_{f}^{2}\left(\lambda_{1}, \lambda_{2}\right)$ given in (2). Thus, the main obstacle is to efficiently and precisely compute the vectors $\omega_{f}(\lambda)$ for a given $\lambda$. This vector is obtained by evaluating the Fourier transform of $f$ on the orbit of $\lambda$ under the action of the rotations $R_{\frac{2 \pi k}{N}}$ for $k \in \mathbb{Z}_{N}$.

Since the Fourier transform $\hat{f}$ of an image is given as a discrete matrix, the usual way to proceed would be to implement rotations as functions on the plane and then evaluate $\hat{f}\left(R_{\frac{2 \pi k}{N}} \lambda\right)$ by bilinear interpolation on the values of $\hat{f}$. However, this requires a lot of matrix products and, especially for values of $\lambda$ very near to 0 where most of the information for natural images is contained, is prone to errors.

We thus chose to consider only $N=6$ and to work with images composed of hexagonal pixels. This choice was motivated by the following reasons:

- It is well-known that retinal cells are distributed in an hexagonal grid.

- Hexagonal grids are invariant under the action of $\mathbb{Z}_{6}$ and discretized translations, which is the most we can get in the line of the invariance w.r.t. $S E(2,6)$.

- We can exploit the Spiral Architecture introduced by Sheridan [16,17]. This is a way to index hexagons of the grid with only one index which allows to introduce an operation, spiral multiplication, that, with the same complexity of a normal multiplication, computes rotations by multiples $\pi / 3$.

- There exist efficient methods [9] to simulate hexagonal pixels by oversampling the image by a ratio of 7 and then using so-called hyperpels composed of 56 pixels to approximate an hexagonal pixel.

\footnotetext{
6 The iPython notebook with the code described in this section is available at http://nbviewer.ipython.org/github/dprn/GSI15/blob/master/ Invariants-computation.ipynb.
} 
Indeed, once the spiral addressing described in [9] has been implemented in the function SpiralAddr and the spiral multiplication in SpiralMult, to evaluate $\omega_{f}(\lambda)$ it suffices to apply Algorithm 3.

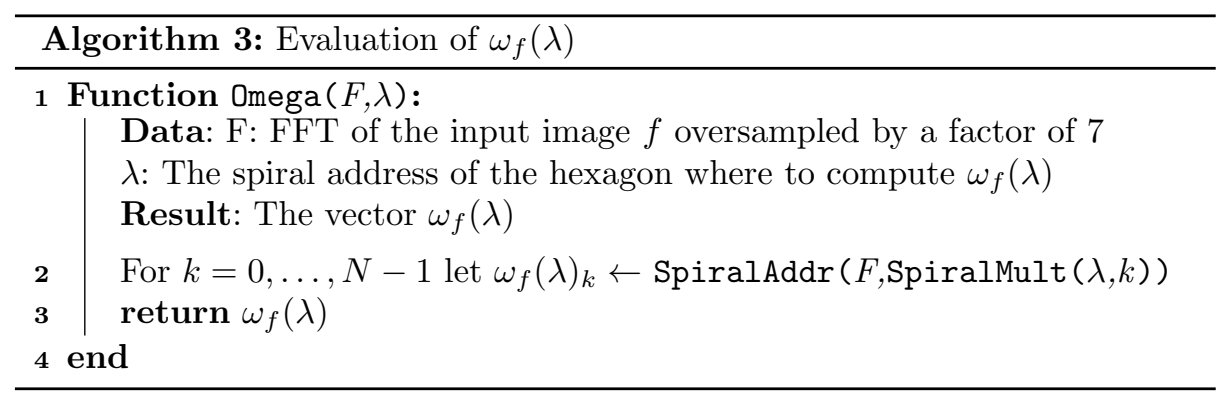

To test the invariants, we built a library composed of 5 geometrical figures rotated of angles $\pi k / 3, k \in \mathbb{Z}_{6}$, and 14 natural images and computed the invariants corresponding to $\lambda_{1}$ and $\lambda_{2}$ chosen between the subset of central hexagons of the grid shown in Figure 2, obtaining a vector $I_{f}^{2}$ of invariants with 49 elements. Then, for each geometrical figure $f$, we computed the difference in norm $\left\|I_{f}^{2}-I_{g}^{2}\right\|$ between its invariants and those of another image $g$, for all the images. In the second and third column of Table 3 we reported the maximal difference w.r.t. the rotated of the same image and the minimal difference w.r.t. the other images. In particular, since the difference between these two values is at least in the order of $10^{2}$, we observe that already simply using the norm seems to be a good discriminating factor for these simple images.

We then repeated the same test with the rotational bispectrum, whose results are reported in the fourth and fifth column of Figure 3. We point out that, accordingly to our conjecture regarding the completeness of the bispectrum, considering the rotational invariants do not seem to add discriminating power.

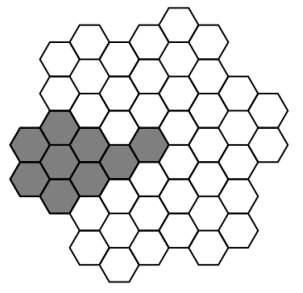

Fig. 2. Hexagons used in the computation of the invariants. The covered area corresponds to roughly 11 square pixels.

\begin{tabular}{|l||c|c|c|c|}
\hline \multicolumn{1}{|c||}{} & \multicolumn{2}{c|}{ bisp. } & \multicolumn{2}{c|}{ rot. bisp. } \\
& Max. & Min & Max. & Min \\
\hline \hline Triangle & $9.2 \times 10^{10}$ & $7.0 \times 10^{12}$ & $2.2 \times 10^{11}$ & $1.7 \times 10^{13}$ \\
\hline Rectangle & $7.9 \times 10^{10}$ & $8.2 \times 10^{12}$ & $1.9 \times 10^{11}$ & $2.0 \times 10^{13}$ \\
\hline Ellipse & $7.4 \times 10^{10}$ & $7.1 \times 10^{12}$ & $1.8 \times 10^{11}$ & $1.7 \times 10^{13}$ \\
\hline Star & $7.2 \times 10^{10}$ & $5.5 \times 10^{12}$ & $1.7 \times 10^{11}$ & $1.3 \times 10^{13}$ \\
\hline Diamond & $3.7 \times 10^{10}$ & $5.4 \times 10^{12}$ & $9.2 \times 10^{10}$ & $1.3 \times 10^{13}$ \\
\hline
\end{tabular}

Fig. 3. Results of the comparisons of the invariants. 


\section{Conclusions}

In this work we presented a framework for image reconstruction and invariant recognition. We remark that the numerical work for the image recognition part has just started. Presently, we are testing the bispectrum as a source of invariant for different machine learning algorithms. In particular, the AdaBoost algorithm seems very promising and well adapted to the problem.

\section{References}

1. Boscain, U., Chertovskih, R., Gauthier, J.P., Remizov, A.: Hypoelliptic diffusion and human vision: a semi-discrete new twist on the petitot theory pp. 1-27 (2013)

2. Boscain, U., Duplaix, J., Gauthier, J.P., Rossi, F.: Anthropomorphic image reconstruction via hypoelliptic diffusion. SIAM J. Control Optim. pp. 1-25 (2012)

3. Boscain, U., Gauthier, J.P., Prandi, D., Remizov, A.: Image reconstruction via non-isotropic diffusion in Dubins/Reed-Shepp-like control systems. In: 53rd IEEE Conference on Decision and Control. pp. 4278-4283 (2014)

4. Boscain, U., Charlot, G., Rossi, F.: Existence of planar curves minimizing length and curvature. Tr. Mat. Inst. Steklova 270(Differentsialnye Uravneniya i Dinamicheskie Sistemy), 49-61 (2010), http://dx.doi.org/10.1134/S0081543810030041

5. Bressloff, P.C., Cowan, J.D., Golubitsky, M., Thomas, P.J., Wiener, M.C.: Geometric visual hallucinations, Euclidean symmetry and the functional architecture of striate cortex. Philos. Trans. R. Soc. Lond. B. Biol. Sci. 356, 299-330 (2001)

6. Citti, G., Sarti, a.: A cortical based model of perceptual completion in the rototranslation space 24(3), 307-326 (2 2006)

7. Dubnov, S., Tishby, N., Cohen, D.: Polyspectra as measures of sound texture and timbre. Journal of New Music Research 26(4), 277-314 (1997)

8. Duits, R., Franken, E.: Left-invariant parabolic evolutions on $\mathrm{SE}(2)$ and contour enhancement via invertible orientation scores Part I: linear left-invariant diffusion equations on SE(2). Quart. Appl. Math. 68(2), 255-292 (2010)

9. He, X., Hintz, T., Wu, Q., Wang, H., Jia, W.: A new simulation of spiral architecture

10. Hewitt, E., Ross, K.: Abstract harmonic analysis - Volume 1. Springer-Verlag (1963)

11. Hladky, R.K., Pauls, S.D.: Minimal surfaces in the roto-translation group with applications to a neuro-biological image completion model. J. Math. Imaging Vision 36(1), 1-27 (2010), http://dx.doi.org/10.1007/s10851-009-0167-9

12. Hubel, D., Wiesel, T.: Receptive fields of single neurones in the cat's striate cortex. J. Physiol. 148, 574-591 (1959)

13. Kakarala, R.: The bispectrum as a source of phase-sensitive invariants for fourier descriptors: a group-theoretic approach (2 2009)

14. Petitot, J.: Neurogéométrie de la vision - Modèles mathématiques et physiques des architectures fonctionnelles. Les Éditions de l'École Polytechnique (2008)

15. Prandi, D., Remizov, A., Chertovskih, R., Boscain, U., Gauthier, J.P.: Highly corrupted image inpainting through hypoelliptic diffusion

16. Sheridan, P.: Spiral Architecture for Machine Vision. Ph.D. thesis (1996)

17. Sheridan, P., Hintz, T., Alexander, D.: Pseudo-invariant image transformations on a hexagonal lattice 18, 907-917 (2000)

18. Smach, F., Lemaître, C., Gauthier, J.p., Miteran, J., Atri, M.: Generalized fourier descriptors with applications to objects recognition in svm context 30, 43-71 (2008) 